\begin{tabular}{|c|l|}
\hline Title & Reciprocal relation between the fractal and the small-world properties of complex networks \\
\hline Author(s) & Kawasaki, F.; Y akubo, K. \\
\hline Citation & $\begin{array}{l}\text { Physical Review E, 82(3), 036113 } \\
\text { https://doi.org/_0.1103/PhysRevE.82.036113 }\end{array}$ \\
\hline Issue Date & 2010-09 \\
\hline Doc URL & http://hdl.handle.net/2115/44003 \\
\hline Rights & O2010 The A merican Physical Society \\
\hline Type & article \\
\hline File Information & PRE82-3_036113.pdf \\
\hline
\end{tabular}

Instructions for use 


\title{
Reciprocal relation between the fractal and the small-world properties of complex networks
}

\author{
F. Kawasaki* and K. Yakubo ${ }^{\dagger}$ \\ Department of Applied Physics, Hokkaido University, Sapporo 060-8628, Japan
}

(Received 23 April 2010; published 24 September 2010)

\begin{abstract}
The fractal and the small-world properties of complex networks are systematically studied both in the box-covering (BC) and the cluster-growing (CG) measurements. We elucidate that complex networks possessing the fractal (small-world) nature in the BC measurement are always fractal (small world) even in the CG measurement and vice versa, while the fractal dimensions $d_{\mathrm{B}}$ by the $\mathrm{BC}$ measurement and $d_{\mathrm{C}}$ by the CG measurement are generally different. This implies that two structural properties of networks, fractality and small worldness, cannot coexist in the same length scale. These properties can, however, crossover from one to the other by varying the length scale. We show that the crossover behavior in a network near the percolation transition appears both in the BC and CG measurements and is scaled by a unique characteristic length $\xi$.
\end{abstract}

DOI: 10.1103/PhysRevE.82.036113

PACS number(s): 89.75.Hc, 64.60.al, 64.60.ah, 64.60.aq

\section{INTRODUCTION}

Numerous complex systems in nature and society can be described by complex networks consisted of nodes and edges [1-3]. It has been shown that many complex networks in the real-world possess common statistical features such as the small-world property [4], the scale-free property [5], and the fractal property [6]. The small-world property is that a network has a short average node-pair distance $l$ increasing logarithmically (or more slowly) with the number of nodes $N$ and a high clustering coefficient. A network is regarded to be scale-free if the degree distribution function obeys a power law. This implies that scale-free networks are highly inhomogeneous and contain a few nodes having very large degrees (hubs). The fractal property represents the self-similar structure of an object without possessing any characteristic length scale. The measure of a conventional fractal object embedded in the Euclidean space is proportional to $l^{D}$, where $l$ is the linear size of the system and $D$ is the (noninteger) fractal dimension. Extending this concept to complex networks, a network is considered to be fractal when the relation $\left\langle M_{\mathrm{C}}\left(l_{\mathrm{C}}\right)\right\rangle \sim l_{\mathrm{C}}^{d_{\mathrm{C}}}$ holds, where $\left\langle M_{\mathrm{C}}\left(l_{\mathrm{C}}\right)\right\rangle$ is the average number of nodes in a subgraph of network diameter $l_{\mathrm{C}}$ and $d_{\mathrm{C}}$ is the fractal dimension of the network measured by the clustergrowing (CG) method. This power-law relation, however, obviously conflicts with the small-world property. In fact, a clear dichotomy between the small-world and the fractal natures has been demonstrated in real-world networks [7].

Despite the apparent inconsistency between two concepts, Song et al. [6] showed that these two properties are reconciled by defining fractality of complex networks in the boxcovering $(\mathrm{BC})$ measurement. In this definition, a network is fractal if the relation $N_{\mathrm{B}}\left(l_{\mathrm{B}}\right) \sim l_{\mathrm{B}}^{-d_{\mathrm{B}}}$ holds, where $N_{\mathrm{B}}\left(l_{\mathrm{B}}\right)$ is the minimum number of boxes (subgraphs) of diameter $l_{\mathrm{B}}$ required to cover the entire network and $d_{\mathrm{B}}$ is the fractal dimension. This definition of fractality is mathematically different from that in the CG measurement, $\left\langle M_{\mathrm{C}}\left(l_{\mathrm{C}}\right)\right\rangle \sim l_{\mathrm{C}}^{d_{\mathrm{C}}}$. Two fractal dimensions $d_{\mathrm{C}}$ and $d_{\mathrm{B}}$ usually take the same value for

\footnotetext{
*fk-1985@eng.hokudai.ac.jp

†yakubo@eng.hokudai.ac.jp
}

conventional fractal objects embedded in the Euclidean spaces, while they can be different for complex networks especially with the scale-free property. If the dimension $d_{\mathrm{B}}$ of a network remains finite but $d_{\mathrm{C}}$ diverges, the network possesses the fractal property in the BC sense and the smallworld nature in the CG sense simultaneously.

Inspired by this idea, fractality of complex networks in the BC measurement has been extensively studied and the coexistence of the fractal and the small-world properties has been supported by many works [6,8-16]. In order to clarify more precisely the relation between fractality and small worldness, several network models with or without the scalefree property have been proposed, in which both of these structural features are provided by controlling parameters $[10,17,18]$. In these models, however, the parameter ranges for fractal networks are separated from those for small-world ones, which implies that fractality even in the $\mathrm{BC}$ sense cannot coexist with the small-world nature in these models. It has also been elucidated that a network shows a crossover behavior from the fractal to the small-world property depending on a length scale if extra shortcut edges are randomly added to a fractal network in the BC sense [19]. Recently, the renormalization approach has been proven to be efficient to extract detailed information on fractality of networks $[20,21]$ and revealed that the crossover behavior and the coarse-grained (renormalized) structure of a network added extra edges strongly depend on how to add shortcut edges to the original fractal network [22]. In the sense of the CG measurement, on the other hand, such a crossover phenomenon has been well argued from the viewpoint of the percolation transition [3,23-25]. Although there exist many works studying the relation between the fractal and the small-world properties of complex networks, it has not yet been clarified whether a fractal network in the BC sense can be nonfractal (small world) by the CG measurement.

In this paper, we examine fractality of several real-world networks and stochastic network models in both senses of the $\mathrm{BC}$ and the $\mathrm{CG}$ measurements, most of which have been studied previously in the context of the fractal nature of networks. Our results suggest that fractal networks in one sense are also fractal even in the other sense though two fractal dimensions $d_{\mathrm{B}}$ and $d_{\mathrm{C}}$ can take different values. This implies that a small-world network never possesses the fractal prop- 

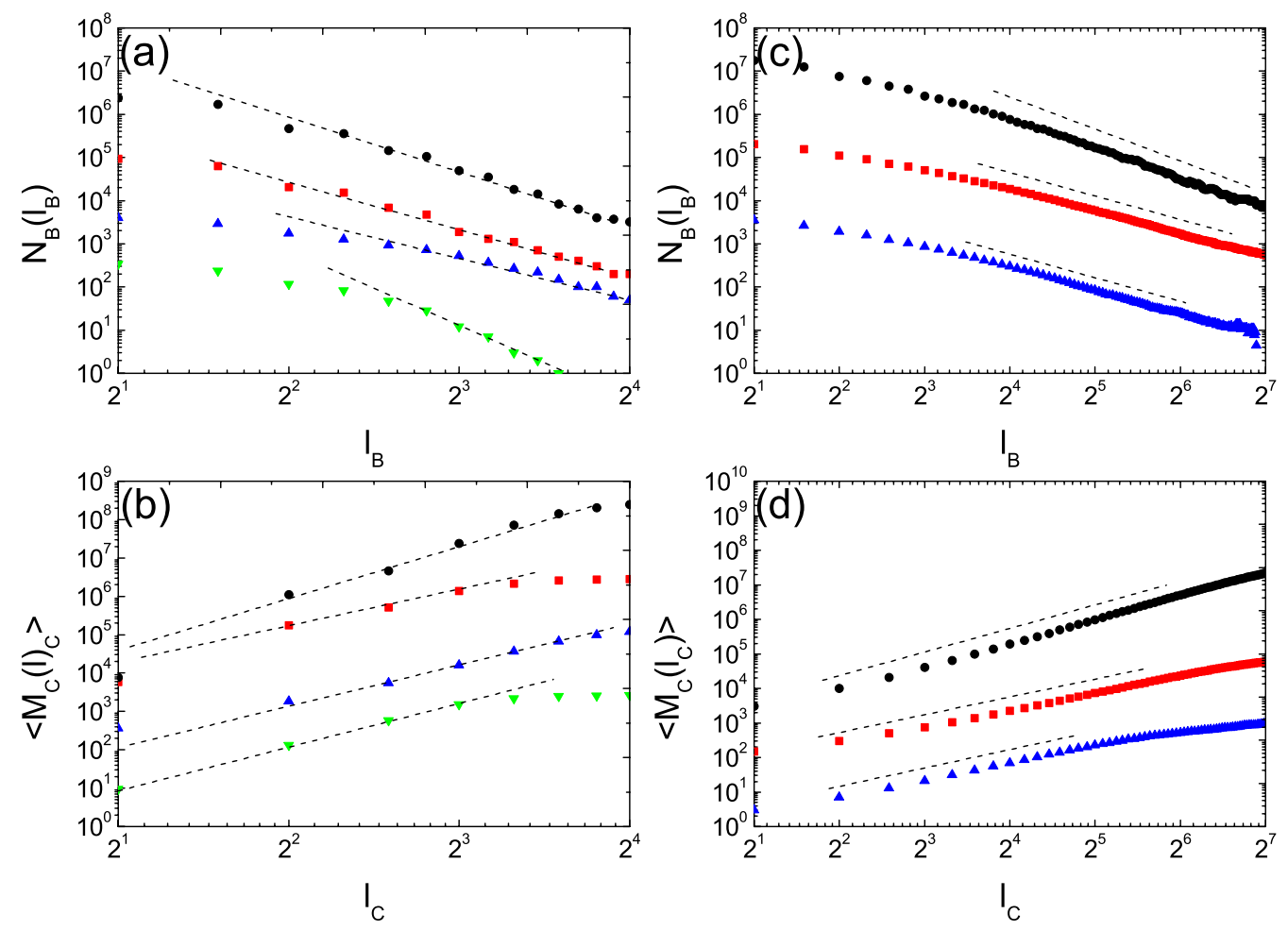

FIG. 1. (Color online) (a) Number of covering boxes as a function of box size $l_{\mathrm{B}}$ for four scale-free networks, namely, from the top to the bottom, the WWW (circles), the cellular network of E. coli (squares), protein interaction networks in yeast (triangles), and H. sapiens (inverted triangles). Dashed lines through marks are a guide to the eyes representing the fractal dimensions of $d_{\mathrm{B}}=4.41$ [7-15] (WWW), 3.55 [3-16] (E. Coli), 3.41 [8-16] (yeast), and 5.12 [5-12] (H. sapiens) estimated by data within the region of $l_{\text {lower }} \leq l_{\mathrm{B}} \leq l_{\text {upper }}$ indicated by $\left[l_{\text {lower }}-l_{\text {upper }}\right]$. (b) Average number of nodes in a box of size $l_{\mathrm{C}}$ for the same networks as treated in (a). The same symbol represents the same network as in (a). Dashed lines represent the fractal dimensions of $d_{\mathrm{C}}=4.44$ [4-14] (WWW), 2.81 [4-10] (E. Coli), 3.74 [6-10] (yeast), and $3.68[2-8]$ (H. sapiens) estimated in the region indicated by $\left[l_{\text {lower }}-l_{\text {upper }}\right]$. (c) Same as (a) for three fractal network models, namely, from the top to the bottom, the SHM model (circles), the ERRG (squares), and the FMN (triangles) at their percolation transition. The fractal dimensions $d_{\mathrm{B}}$ represented by dashed lines are $d_{\mathrm{B}}=2.67$ (SHM), 1.77 (ERRG), and 1.84 (FMN). (d) Same as (b) for the same networks as treated in (c). The same symbol represents the same network as in (c). Dashed line through marks represent $d_{\mathrm{C}}=2.34(\mathrm{SHM}) 1.70(\mathrm{ERRG})$, and $1.82(\mathrm{FMN})$. In these figures, the data are shifted vertically for clarity.

erty either in the BC and the CG senses. Furthermore, we demonstrate that a network above the percolation transition exhibits a crossover phenomenon from the fractal to the small-world property not only in the CG sense but also in the $\mathrm{BC}$ sense. Our result elucidates that the crossover behavior measured by the $\mathrm{BC}$ method is scaled by a unique characteristic length as in the case of the CG measurement.

The rest of this paper is organized as follows. In Sec. II, we investigate fractality, in the $\mathrm{CG}$ sense, of real-world networks known to be fractal in the BC sense. The small-world property (in the CG sense) of some fractal network models is also examined in this section. In Sec. III, the crossover behavior from the fractal to the small-world nature is argued in the $\mathrm{BC}$ and the $\mathrm{CG}$ senses from the viewpoint of the percolation transition of networks. We present our conclusion in Sec. IV.

\section{FRACTALITY IN THE BC AND CG SENSES}

It has been reported that a World Wide Web (WWW) network takes a fractal structure in the BC sense [6] while the small-world property has also been observed by the CG measurement [26], which indicates the coexistence of two structural features. We can find similar examples of the coexistence in power-grid networks $[4,7]$ and software networks $[27,28]$. In such examples, however, scales in measurements are different or, in some cases, a network exhibiting fractality is not identical to a network providing the small-world nature. Here, we systematically examine, both in the $\mathrm{BC}$ and $\mathrm{CG}$ measurements, structures of several complex networks ever treated in the literature.

First, we examine four real-world scale-free networks, namely, the WWW of $N=325729$ nodes and $m=1469680$ edges [29], the cellular network of $E$. coli $(N=2859, m$ $=7104$ ) [29], protein interaction networks (PINs) of the yeast $(N=1458, m=2203)$ [29], and H. sapiens $(N=1522, m$ $=2611$ ) $[30,31]$. These networks are known to be fractal in the $\mathrm{BC}$ sense $[6,8]$. The number of boxes required to cover the network is plotted in Fig. 1(a) for these four networks as a function of the box size $l_{\mathrm{B}}$. To achieve the minimum covering, we employed the compact-box-burning algorithm [32] modified to shorten the computing time [33]. Figure 1(b) shows the average number of nodes within a box of size $l_{\mathrm{C}}$ for the same networks. Results in Fig. 1(b) suggest that all these networks shown to be fractal by the BC measurement 
are also fractal even in the CG measurement. Since diameters of these networks are not sufficiently large, it is not easy to determine definitely whether a network is fractal or small world. It is, however, possible to find fractal scaling regimes in Fig. 1(b) at least for small $l_{\mathrm{C}}$. The fractal dimensions $d_{\mathrm{C}}$ of these networks are not very different from $d_{\mathrm{B}}$ except for the PIN of H. sapiens. It should be noted that $\left\langle M_{\mathrm{C}}\left(l_{\mathrm{C}}\right)\right\rangle$ in Fig. 1(b) does not obey the fractal scaling at a longer length scale than $2^{3}$ while the behavior of $N_{\mathrm{B}}\left(l_{\mathrm{B}}\right)$ still indicates fractality in this scale. This is due to the finite-size effect peculiar to scale-free networks. When $l_{\mathrm{C}}$ approaches the network diameter, a box includes a huge hub and almost all of nodes in the network are contained in this box. As a result, the quantity $\left\langle M_{\mathrm{C}}\left(l_{\mathrm{C}}\right)\right\rangle$ is not sensitive to $l_{\mathrm{C}}$. Thus, the fractal scaling regime by the $\mathrm{CG}$ method is usually narrower than that by the $\mathrm{BC}$ method for scale-free networks. In a network with a relatively small diameter, this effect becomes serious to find the fractal property. Therefore, the BC method is more appropriate to study fractality of scale-free networks. However, we should note that the difficulty to find the fractal property in the CG measurement does not imply that the network is intrinsically small world in the CG sense.

Next, we examine structures of complex networks constructed by three stochastic models. At first, we study the Song-Havlin-Makse (SHM) model [8], in which a network is formed by an inverse procedure of the renormalization scheme and possesses the scale-free property. The SHM model has an important parameter $e(\in[0,1])$ controlling the degree of assortativity (attractive correlation between hubs). The network becomes fractal or small world at $e=0$ (disassortative) or $e=1$ (assortative), respectively. We measure $N_{\mathrm{B}}\left(l_{\mathrm{B}}\right)$ and $\left\langle M_{\mathrm{C}}\left(l_{\mathrm{C}}\right)\right\rangle$ for a network made by the SHM model at $e=0$ [34]. The network has 46656 nodes and 46655 edges. Results are shown by circles in Figs. 1(c) and 1(d). As in the case of real-world networks, the fractal property of the network is found both in the $\mathrm{BC}$ and the $\mathrm{CG}$ measurements. The fractal dimension $d_{\mathrm{B}}$ is close to the theoretical prediction $d_{\mathrm{B}}=2.58$ [8]. It should be noted that the fractal dimension $d_{\mathrm{C}}$ is not very different from $d_{\mathrm{B}}$ though the SHM network has the scale-free property with the degree exponent of $\gamma=3.58$. It has been pointed out that there is a possibility of a fractal small-world network at an intermediate value of the assortativity parameter $e$ [8]. In order to inspect this possibility, we calculate $N_{\mathrm{B}}\left(l_{\mathrm{B}}\right)$ and $\left\langle M_{\mathrm{C}}\left(l_{\mathrm{C}}\right)\right\rangle$ for SHM networks with $e=0.2$ and 0.8 . The results are presented in Fig. 2. For $e=0.2$, the network is well approximated to be fractal in both senses of $\mathrm{BC}$ and $\mathrm{CG}$. On the contrary, these quantities, $N_{\mathrm{B}}\left(l_{\mathrm{B}}\right)$ and $\left\langle M_{\mathrm{C}}\left(l_{\mathrm{C}}\right)\right\rangle$, for $e=0.8$ show exponential behavior, which implies the small-world property of the network. Therefore, our numerical results suggest a reciprocal relation between the fractal and the small-world properties in the SHM model.

Other networks by stochastic models we examine are realized as critical networks in two kinds of percolation processes. One is the Erdős-Rényi random graph (ERRG) in which every node pair among $N$ nodes is connected with a given probability $p[35]$. For the ERRG, the percolation transition occurs at $p=1 / N\left(=p_{\mathrm{c}}\right)$ above which the number of nodes in the largest cluster (giant component) becomes proportional to $N[35]$. It has been clarified that the largest clus-

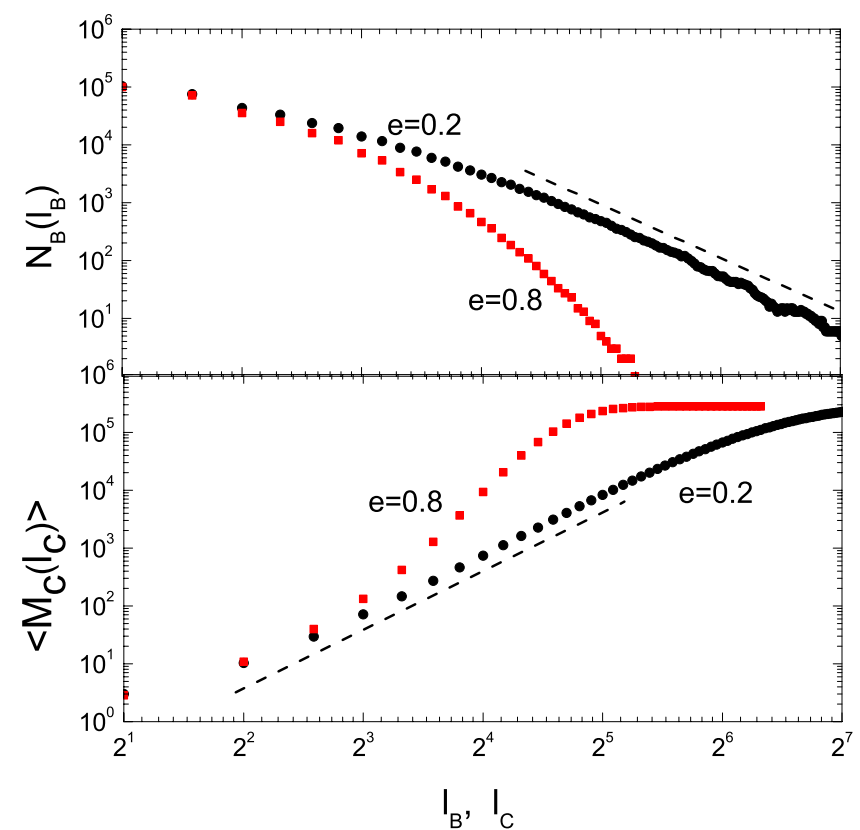

FIG. 2. (Color online) The upper panel shows the number of covering boxes as a function of box size $l_{\mathrm{B}}$ for the SHM model with $e=0.2$ (circle) and 0.8 (square). The lower panel shows the average number of nodes in a box of size $l_{\mathrm{C}}$ as a function of $l_{\mathrm{C}}$ for the same networks. Dashed lines are a guide to the eyes with the slopes of $d_{\mathrm{B}}=3.19$ and $d_{\mathrm{C}}=3.44$.

ter of the ERRG at the percolation threshold takes a fractal structure in the CG sense [23] and the BC sense [6]. We confirm this fact as shown in Figs. 1(c) and 1(d). Here, the total number of nodes is $N=100000$ and the results are averaged over 100 realizations. Due to the lack of the scale-free property in the ERRG, the fractal scaling in $\left\langle M_{\mathrm{C}}\left(l_{\mathrm{C}}\right)\right\rangle$ holds even for a large $l_{\mathrm{C}}$. The fractal dimensions $d_{\mathrm{B}}$ and $d_{\mathrm{C}}$ of the non-scale-free ERRG are expected to be the same. Our numerical results of these two fractal dimensions are surely close to each other but slightly smaller than the mean-field prediction $d_{\mathrm{B}}=d_{\mathrm{C}}=2$ [23]. This can also be seen in the result in Ref. [6] and might be caused by a finite-size effect in numerical calculations. Although results of the ERRG presented here have been already clarified and do not provide any new insights, we display them for comparison with results for scale-free networks.

Another example of critical networks is provided by the fitness model [36-38]. In this model, we start with $N$ isolated nodes and attribute randomly a fitness to each node. The fitness $f_{i}$ of the node $i$ taking a real continuous value in the range of $[1, \infty)$ obeys the power-law distribution

$$
\rho(f)=(\gamma-1) f^{-\gamma},
$$

where $\gamma(>2)$ is an exponent. Every node pair $(i, j)$ is then connected with the probability

$$
p_{i j}=\theta\left(\alpha \frac{f_{i} f_{j}}{N}\right),
$$

where $\alpha$ is a parameter and the function $\theta(x)$ is defined as $\theta(x)=x$ for $x<1$ and $\theta(x)=1$ otherwise. In the case that the 
number of node pairs satisfying $\alpha f_{i} f_{j} / N>1$ is much smaller than $N(N-1) / 2$, the fitness-model network (FMN) has the scale-free property in a large degree regime with the degree exponent equal to the fitness distribution exponent $\gamma[36]$. In our numerical calculations, the exponent $\gamma$ is fixed as $\gamma$ $=4.0$. Then, the FMN is characterized only by $\alpha$. The FMN changes its structure from the set of isolated nodes $(\alpha=0)$ to the complete graph $(\alpha=N)$ gradually by varying the parameter $\alpha$. Thus, we have the percolation transition at a certain value of $\alpha$. Since the linking probabilities $p_{i j}$ are independent to each other and the number of loops in an FMN near the transition point is negligibly small, the percolation threshold $\alpha_{\mathrm{c}}$ is given by the relation

$$
\sum_{k} k(k-2) P_{\alpha_{\mathrm{c}}}^{\gamma}(k)=0
$$

where $P_{\alpha}^{\gamma}(k)$ is the degree distribution function of the FMN for the parameter $\gamma$ and $\alpha$ [39-43]. We numerically calculated the entire profile of $P_{\alpha}^{\gamma}(k)$ and obtained $\alpha_{\mathrm{c}}$ $=0.34 \pm 0.01$ at $\gamma=4.0$ by using Eq. (3) and extrapolating to the thermodynamic limit $(N \rightarrow \infty)$. Triangles in Figs. 1(c) and $1(\mathrm{~d})$ indicate $N_{\mathrm{B}}\left(l_{\mathrm{B}}\right)$ and $\left\langle M_{\mathrm{C}}\left(l_{\mathrm{C}}\right)\right\rangle$ averaged over 100 realizations of FMNs $(N=50000)$ at $\alpha=\alpha_{\mathrm{c}}$. These results clearly show that the scale-free FMN at the percolation threshold exhibits the fractal property both in the $\mathrm{BC}$ and the $\mathrm{CG}$ senses. It is interesting to note that the fractal dimensions $d_{\mathrm{B}}$ and $d_{\mathrm{C}}$ of the scale-free FMN take almost the same value as in the case of the non-scale-free ERRG.

All these results definitely demonstrate that a fractal network in the BC sense is also fractal even in the CG sense regardless of the scale-free property of the network and vice versa. This implies that the fractal property of a network cannot coexist with the small-world nature in the same length scale independently of the measurement method, which contradicts the previous views in the literature $[6,8-16]$. Although the fractal property can be easily detected by the $\mathrm{BC}$ method rather than the $\mathrm{CG}$ method, the intrinsic structural property of a network is irrelevant to the way of measurement. This conclusion seems to be reasonable if we consider a characteristic length of a network. A small-world network described by $\left\langle M_{\mathrm{C}}\left(l_{\mathrm{C}}\right)\right\rangle \sim e^{l_{C} l_{0}}$ must include the characteristic length $l_{0}$ while a fractal network must not. The existence (absence) of such a characteristic length never depends on how we measure the network structure. Here, we consider two fractal dimensions $d_{\mathrm{B}}$ and $d_{\mathrm{C}}$. These fractal dimensions take close values to each other for each of all empirical and algorithmic networks we treated, except for the PIN of H. sapiens. Since the definitions of $d_{\mathrm{B}}$ and $d_{\mathrm{C}}$ are mathematically independent, these fractal dimensions are not necessary to have the same value. The fractal dimension $d_{\mathrm{C}}$ represents dominantly the fractal property near hub nodes because the CG method counts many times measures near hubs, while $d_{\mathrm{B}}$ reflects fractality uniformly the entire network. If the local fractal dimension near hubs is different from that apart from hubs, $d_{\mathrm{C}}$ might be different from $d_{\mathrm{B}}$ as in the case of the PIN of H. sapiens. However, further investigations are required to elucidate more clearly the condition for $d_{\mathrm{B}}=d_{\mathrm{C}}$. It should be emphasized that our claim on the equivalence of fractality both in the $\mathrm{BC}$ and $\mathrm{CG}$ senses is independent of the problem of whether $d_{\mathrm{B}}$ is equal to $d_{\mathrm{C}}$.

\section{CROSSOVER FROM FRACTAL TO SMALL-WORLD BEHAVIOR}

Our conclusion in Sec. II suggests that the apparent coexistence of the small-world and the fractal properties observed in several real-world networks $[4,6,7,26-28]$ is not explained by the difference in measurement methods. A possible explanation of such a coexistence is given by a structural crossover depending on the length scale. The crossover behavior from a fractal to small-world structure has been found by adding shortcut edges to a fractal scale-free network formed by the SHM model [19]. In addition, it has been well established that in the sense of the CG measurement a network above the percolation transition shows the crossover between the fractal and the small-world properties and the crossover behavior can be scaled by the correlation length $\xi[23,24]$. Since the presence of fractality does not depend on the measurement method, the scaling property of the crossover behavior near the percolation transition is expected to be irrelevant to whether the network is analyzed in the $\mathrm{BC}$ or $\mathrm{CG}$ measurement. In this section, we demonstrate the above issue by examining $N_{\mathrm{B}}\left(l_{\mathrm{B}}\right)$ and $\left\langle M_{\mathrm{C}}\left(l_{\mathrm{C}}\right)\right\rangle$ of non-scale-free and scale-free networks slightly above their percolation transition points.

We numerically calculated $N_{\mathrm{B}}\left(l_{\mathrm{B}}\right)$ and $\left\langle M_{\mathrm{C}}\left(l_{\mathrm{C}}\right)\right\rangle$ for ERRGs and FMNs at and above their percolation thresholds. Conditions for calculations are the same as those for Fig. 1 except for $p$ and $\alpha$. Results are shown in Figs. 3 and 4 for ERRGs and FMNs, respectively. The ERRG and FMN at criticality take fractal structures in a wide range of length scale both in the BC and $\mathrm{CG}$ measurements. As shown in Figs. 3(a) and 4(a), $N_{\mathrm{B}}\left(l_{\mathrm{B}}\right)$ for $p>p_{c}\left(\alpha>\alpha_{c}\right)$ deviates from a power-law form for large $l_{\mathrm{B}}$. This implies that additional shortcut edges to the fractal network at the critical point make the network small world in a long scale. Similar crossover behaviors from fractal to small-world structure are found also in the CG measurement as depicted in Figs. 3(b) and 4(b). In both measurements, the crossover length scale decreases as getting away from the percolation transition point. Since the ERRG and the FMN are a non-scale-free and scale-free network, respectively, the crossover behavior is irrelevant not only to the measurement method but also to the scale-free property.

In order to confirm that the crossover behaviors found in $N_{\mathrm{B}}\left(l_{\mathrm{B}}\right)$ and $\left\langle M_{\mathrm{C}}\left(l_{\mathrm{C}}\right)\right\rangle$ come from the same physical origin, we performed the scaling analysis for these data. Assuming that both $N_{\mathrm{B}}\left(l_{\mathrm{B}}\right)$ and $\left\langle M_{\mathrm{C}}\left(l_{\mathrm{C}}\right)\right\rangle$ near the critical point are scaled by the correlation length $\xi \sim\left|z-z_{\mathrm{c}}\right|^{-\nu}$, where $z$ denotes $p$ or $\alpha$ for the ERRG or the FMN, respectively, and $\nu$ is the correlation length exponent, we have

$$
\frac{N_{\mathrm{B}}\left(l_{\mathrm{B}}\right)}{N_{\mathrm{cl}}}=\left(z-z_{\mathrm{c}}\right)^{\nu d_{\mathrm{B}}} F\left[\left(z-z_{\mathrm{c}}\right) l_{\mathrm{B}}^{1 / \nu}\right]
$$



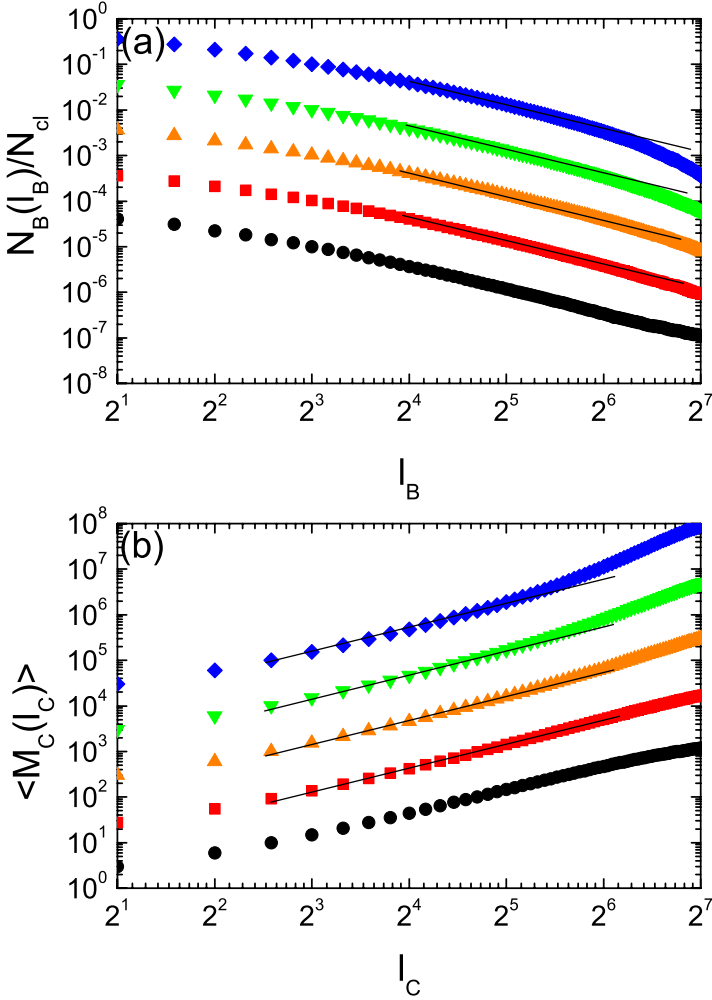

FIG. 3. (Color online) (a) Number of covering boxes as a function of box size $l_{\mathrm{B}}$ for the Erdős-Rényi random graphs of $N$ $=100000$ rescaled by the size of the giant component. Solid lines are a guide to the eyes with the slope of $d_{\mathrm{B}}=1.77$. (b) Average number of nodes in a box of size $l_{\mathrm{C}}$ as a function of $l_{\mathrm{C}}$ for the same networks. Results are averaged over 100 realizations. Solid lines are a guide to the eyes with the slope of $d_{C}=1.70$. Different symbols represent results for different probabilities, $p=p_{\mathrm{c}}$ (circle), $p$ $=1.025 p_{\mathrm{c}}$ (square), $p=1.05 p_{\mathrm{c}}$ (triangle), $p=1.075 p_{\mathrm{c}}$ (inverse triangle), and $p=1.1 p_{\mathrm{c}}$ (diamond). In both figures, the data are shifted vertically for clarity.

$$
\left\langle M_{\mathrm{C}}\left(l_{\mathrm{C}}\right)\right\rangle=\left(z-z_{\mathrm{c}}\right)^{-\nu d_{\mathrm{C}}} G\left[\left(z-z_{\mathrm{c}}\right) l_{\mathrm{C}}^{1 / \nu}\right],
$$

where $N_{\mathrm{cl}}$ is the number of nodes in the giant component. Figure 5 shows the quantities $N_{\mathrm{B}}\left(l_{\mathrm{B}}\right)\left(z-z_{\mathrm{c}}\right)^{-\nu d_{\mathrm{B}} / N_{\mathrm{cl}}}$ and $\left.\left\langle M_{\mathrm{C}}\left(l_{\mathrm{C}}\right)\right\rangle\left(z-z_{\mathrm{c}}\right)\right)^{\nu d_{\mathrm{C}}}$ as functions of $\left(z-z_{\mathrm{c}}\right) l_{\mathrm{B}}^{1 / \nu}$ and $\left(z-z_{\mathrm{c}}\right) l_{\mathrm{C}}^{1 / \nu}$, respectively, for ERRGs and FMNs. The fact that all data in Figs. 3 and 4 collapse onto unique curves supports the validity of the above scaling forms. In the rescaling procedure for ERRGs [Figs. 5(a) and 5(b)], we used values of $d_{\mathrm{B}}$ and $d_{\mathrm{C}}$ obtained numerically from Fig. 1 and $\nu=1$ that is theoretically predicted for the ERRG $[3,44]$. In the case of FMNs [Figs. 5(c) and 5(d)], we employed values of $d_{\mathrm{B}}$ and $d_{\mathrm{C}}$ obtained numerically from Fig. 1 and treated $\nu$ as a fitting parameter. The exponent $\nu$ is then evaluated as $\nu=0.28$ for $N_{\mathrm{B}}\left(l_{\mathrm{B}}\right)$ and $\nu=0.23$ for $\left\langle M_{\mathrm{C}}\left(l_{\mathrm{C}}\right)\right\rangle$, which are close to each other. As shown in Fig. 5, $N_{\mathrm{B}}\left(l_{\mathrm{B}}\right)$ and $\left\langle M_{\mathrm{C}}\left(l_{\mathrm{C}}\right)\right\rangle$ for scale-free and non-scale-free networks above the percolation threshold are scaled by the unique correlation length $\xi \sim\left|z-z_{\mathrm{c}}\right|^{-\nu}$ with the same exponent $\nu$ for $N_{\mathrm{B}}\left(l_{\mathrm{B}}\right)$ and $\left\langle M_{\mathrm{C}}\left(l_{\mathrm{C}}\right)\right\rangle$. This implies that the same physical origin causes the crossover behavior appearing in the $\mathrm{BC}$ and the $\mathrm{CG}$ measurements, though the
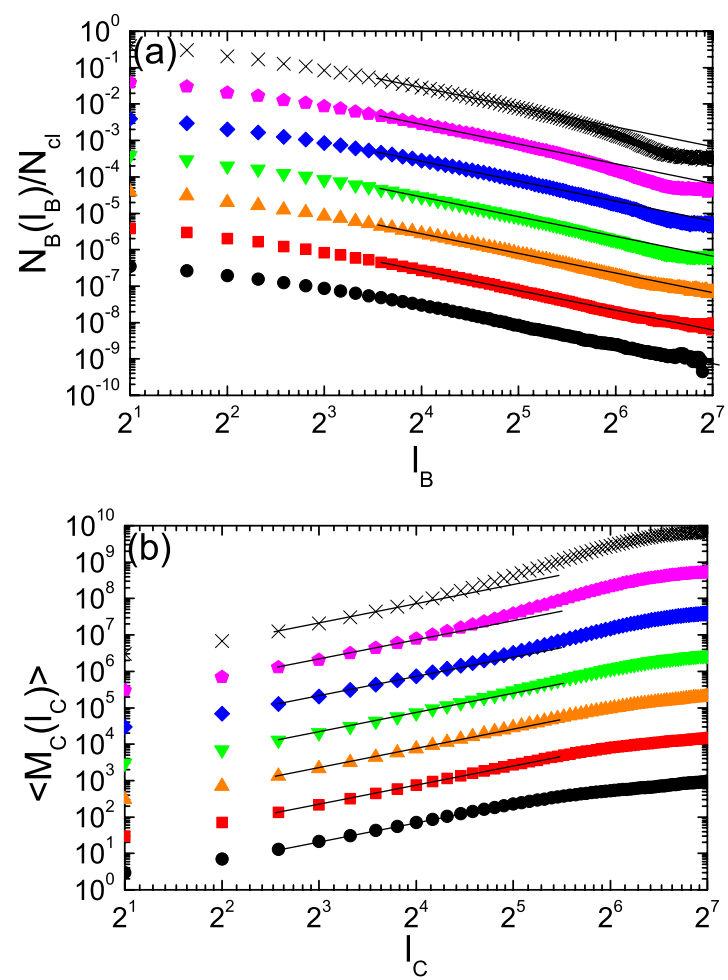

FIG. 4. (Color online) (a) Number of covering boxes as a function of box size $l_{\mathrm{B}}$ for the fitness-model networks of $N=50000$ rescaled by the size of the giant component. Solid lines are a guide to the eyes with the slope of $d_{\mathrm{B}}=1.84$. (b) Average number of nodes in a box of size $l_{\mathrm{C}}$ as a function of $l_{\mathrm{C}}$ for the same networks. Results are averaged over 100 realizations. Solid lines are a guide to the eyes with the slope of $d_{\mathrm{C}}=1.82$. Different symbols represent results for various different of $\alpha, \alpha=\alpha_{\mathrm{c}}=0.34$ (circle), $\alpha=1.03 \alpha_{\mathrm{c}}$ (square), $\alpha=1.06 \alpha_{\mathrm{c}}$ (triangle), $\alpha=1.09 \alpha_{\mathrm{c}}$ (inverse triangle), $\alpha=1.12 \alpha_{\mathrm{c}}$ (diamond), $\alpha=1.15 \alpha_{\mathrm{c}}$ (pentagon), and $\alpha=1.18 \alpha_{\mathrm{c}}$ (cross). In both figures, the data are shifted vertically for clarity.

crossover length by the CG measurement seems to be larger than that by the BC method due to the finite-size effect as shown in Figs. 3 and 4.

We should note that the crossover phenomenon in a complex network near the percolation transition is essentially the same as the dimensional crossover from fractal to Euclidean structure in the percolation problem of a regular lattice [44]. Only the difference is that the dimension of the network at one stable fixed point $p=1$ is infinite (namely, small world) while the dimension of the perfect lattice realized at $p=1$ is finite.

\section{CONCLUSIONS}

We have studied the fractal and the small-world properties of real-world complex networks and several network models in the $\mathrm{BC}$ and the $\mathrm{CG}$ measurements. In contrast to previous studies, all of our numerical analyses suggest that a fractal (small-world) network in one measurement is also fractal (small world) even in another measurement. This implies that these structural features of networks do not depend on the measurement method and cannot coexist in the same 

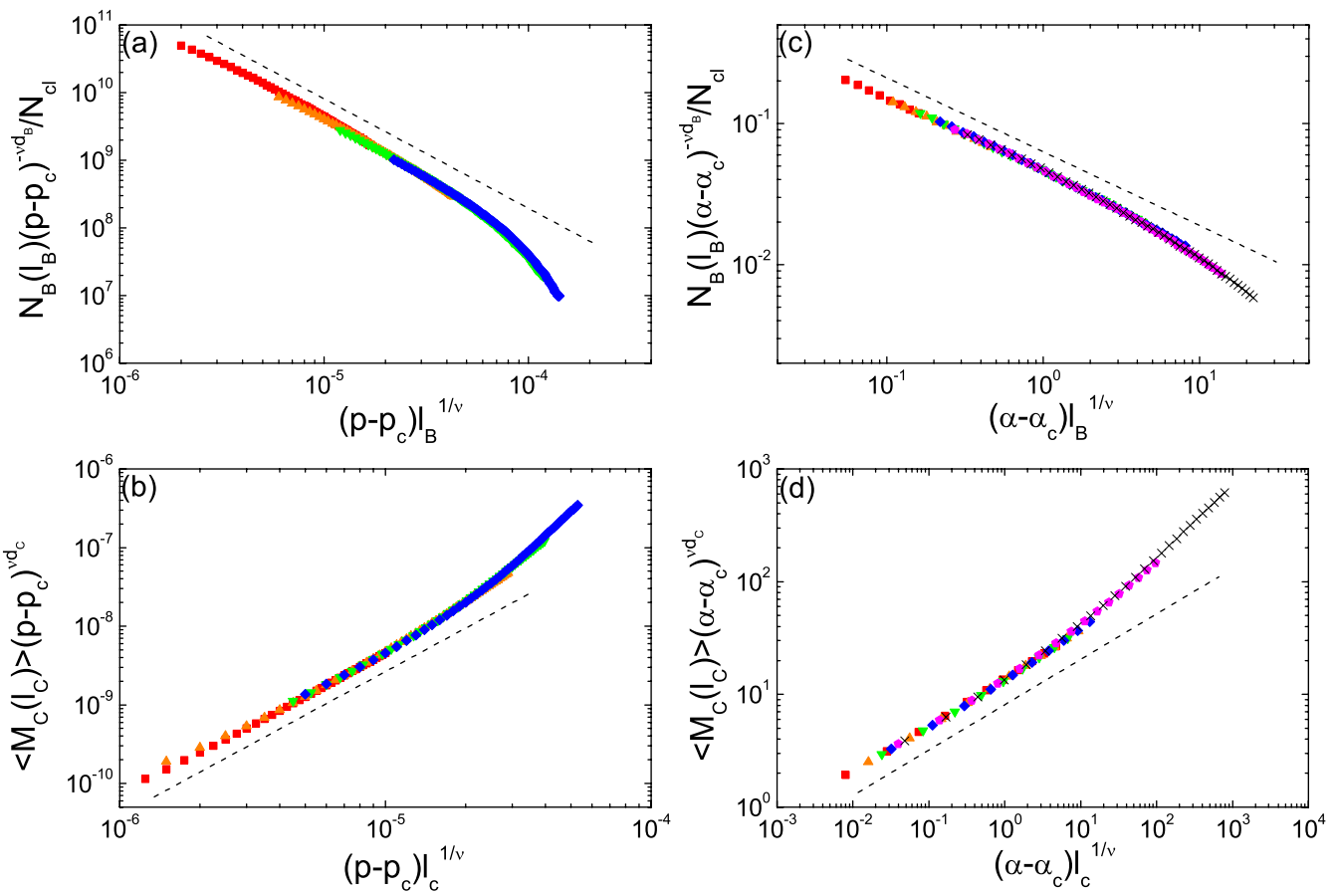

FIG. 5. (Color online) (a) Scaling plot of the ERRG data presented in Fig. 3(a). Dashed line is guide to the eyes representing the fractal scaling with $d_{\mathrm{B}}=1.77$. (b) Scaling plot of the ERRG data presented in Fig. 3(b). Dashed line represents the fractal scaling with $d_{\mathrm{C}}=1.70$. (c) Same as (a) for the FMN data presented in Fig. 4(a). Dashed line represents the fractal scaling with $d_{\mathrm{B}}=1.84$. (d) Same as (b) for the FMN data presented in Fig. 4(b). Dashed line represents the fractal scaling with $d_{C}=1.82$. Meanings of symbols in these figures are the same as those in corresponding Fig. 3 or 4.

length scale. A network can, however, exhibit the structural crossover from fractal to small-world scaling by varying the length scale. Examining the Erdős-Rényi random graphs and fitness-model networks above their percolation transition points, it has been found that such a crossover behavior appears both in the $\mathrm{BC}$ and the $\mathrm{CG}$ measurements. In addition, our scaling analysis reveals that the crossover behaviors in $N_{\mathrm{B}}\left(l_{\mathrm{B}}\right)$ and $\left\langle M_{\mathrm{C}}\left(l_{\mathrm{C}}\right)\right\rangle$ are caused by the same physical origin.

Most of the real-world complex networks show the smallworld property. More precisely, the average node-pair distance $\langle l\rangle$ (or the network diameter) is much smaller than the number of nodes $N$. This fact can be reasonably understood from the viewpoint of the crossover behavior near the percolation transition. A large-scale network usually approaches to the critical network by removing edges randomly [23], which means that the network is above the percolation threshold. Otherwise the network size $N$ remains small. Since the quantity $\langle l\rangle$ is averaged over all node pairs in the network, $\langle l\rangle$ reflects the network structure at the length scale of the diameter. Therefore, in most of the networks, we detect the smallworld property by measuring $\langle l\rangle$. If we observe $N_{\mathrm{B}}\left(l_{\mathrm{B}}\right)$ or $\left\langle M_{\mathrm{C}}\left(l_{\mathrm{C}}\right)\right\rangle$ in a wide range of real-world networks, we might find the fractal nature in a short-length scale unless networks are far beyond the percolation threshold.

\section{ACKNOWLEDGMENTS}

We are grateful to Y. Oka for helpful discussions. This work was supported in part by a Grant-in-Aid for Scientific Research (Grants No. 19560001 and No. 19360042) and Grant-in-Aid for JSPS Fellows (Grant No. 21003752) from Japan Society for the Promotion of Science. Numerical calculations in this work were performed on the facilities of the Supercomputer Center, Institute for Solid State Physics, University of Tokyo.
[1] D. R. Albert and A.-L. Barabási, Rev. Mod. Phys. 74, 47 (2002).

[2] S. N. Dorogovtsev and J. F. F. Mendes, Evolution of Networks: From Biological Nets to the Internet and WWW (Oxford University Press, Oxford, 2003).

[3] Handbook of Graphs and Networks: From the Genome to the Internet, edited by S. Bornholdt and H. G. Schuster (WILEYVCH, Weinheim, 2003).
[4] D. J. Watts and S. H. Strogatz, Nature (London) 393, 440 (1998).

[5] A.-L. Barabási and R. Albert, Science 286, 509 (1999).

[6] C. Song, S. Havlin, and H. A. Makse, Nature (London) 433, 392 (2005).

[7] G. Csányi and B. Szendröi, Phys. Rev. E 70, 016122 (2004).

[8] C. Song, S. Havlin, and H. A. Makse, Nat. Phys. 2, 275 (2006). 
[9] L. Barrière, F. Comellas, and C. Dalfó, J. Phys. A 39, 11739 (2006).

[10] Z. Z. Zhang, S. G. Zhou, L. C. Chen, and J. Guan, Eur. Phys. J. B 64, 277 (2008).

[11] M. Guida and F. Maria, Chaos, Solitons Fractals 31, 527 (2007).

[12] J. S. Kim, K.-I. Goh, G. Salvi, E. Oh, B. Kahng, and D. Kim, Phys. Rev. E 75, 016110 (2007).

[13] J. S. Kim, K.-I. Goh, B. Kahng, and D. Kim, New J. Phys. 9, 177 (2007).

[14] J. S. Kim, K.-I. Goh, B. Kahng, and D. Kim, Chaos 17, 026116 (2007).

[15] L. H. Zou, W. J. Pei, T. Lia, Z. Hea, and Y. Cheung, Physica A 380, 592 (2007).

[16] L. K. Gallos, C. M. Song, and H. A. Makse, Physica A 386, 686 (2007)

[17] S. Carmi, P. L. Krapivsky, and D. ben-Avraham, Phys. Rev. E 78, 066111 (2008).

[18] R. F. S. Andrade, J. G. V. Miranda, S. T. R. Pinho, and T. P. Lobao, Eur. Phys. J. B 61, 247 (2008).

[19] M. Kitsak, S. Havlin, G. Paul, M. Riccaboni, F. Pammolli, and H. E. Stanley, Phys. Rev. E 75, 056115 (2007).

[20] F. Radicchi, J. J. Ramasco, A. Barrat, and S. Fortunato, Phys. Rev. Lett. 101, 148701 (2008).

[21] F. Radicchi, A. Barrat, S. Fortunato, and J. J. Ramasco, Phys. Rev. E 79, 026104 (2009).

[22] H. D. Rozenfeld, C. Song, and H. A. Makse, Phys. Rev. Lett. 104, 025701 (2010).

[23] R. Cohen, K. Erez, D. ben-Avraham, and S. Havlin, Phys. Rev. Lett. 86, 3682 (2001).

[24] R. Cohen and S. Havlin, Physica A 336, 6 (2004).

[25] S. N. Dorogovtsev and A. V. Goltsev, Rev. Mod. Phys. 80, 1275 (2008).

[26] R. Albert, H. Jeong, and A.-L. Barabási, Nature (London) 401, 130 (1999).

[27] G. Concas, M. F. Locci, M. Marchesi, S. Pinna, and I. Turnu, Europhys. Lett. 76, 1221 (2006).

[28] C. R. Myers, Phys. Rev. E 68, 046116 (2003).

[29] Center for Complex Network Research (CCNR), http:// www.nd.edu/networks/resources.htm
[30] Center for Cancer Systems Biology (CCSB), http:// ccsb.dfci.harvard.edu/web/www/ccsb/

[31] We should note that the network data set for the PIN of $\mathrm{H}$. sapiens is different from that used in Ref. [6]. Therefore, the fractal dimension of this network differs from that reported in Ref. [6].

[32] C. Song, L. K. Gallos, S. Havlin, and H. A. Makse, J. Stat. Mech.: Theory Exp. (2007), P03006.

[33] Although we first cover high degree hubs for reducing the computing time, the fractal property obtained by this method characterizes the entire structure of the network without removing hubs.

[34] The SHM model has actually four parameter, $m, n, a$, and $g$. Meanings of these parameters are the ratio of the number of nodes in the renormalized graph to that in the prerenormalized one $(m)$, the ratio of the degree of the renormalized node to that of the prerenormalized node $(n)$, the ratio of the diameter of the renormalized network to that of the prerenormalized network $(a)$, and the generation $(g)$. We choose $m=2, n=6$, $a=2$, and $g=6$ for Figs. 1 (c) and $1(\mathrm{~d})$ and $m=2, n=6, a=2$, and $g=7$ for Fig. 2.

[35] P. Erdős and A. Rényi, Publ. Math. (Debrecen) 6, 290 (1959).

[36] G. Caldarelli, A. Capocci, P. De Los Rios, and M. A. Munõz, Phys. Rev. Lett. 89, 258702 (2002).

[37] D. Garlaschelli, A. Capocci, and G. Caldarelli, Nat. Phys. 3, 813 (2007).

[38] M. Mitobe and K. Yakubo, J. Phys. Soc. Jpn. 78, 124002 (2009).

[39] M. Molloy and B. Reed, Random Struct. Algorithms 6, 161 (1995).

[40] M. Molloy and B. Reed, Combinatorics, Probab. Comput. 7, 295 (1998).

[41] R. Cohen, K. Erez, D. ben-Avraham, and S. Havlin, Phys. Rev. Lett. 85, 4626 (2000).

[42] D. S. Callaway, M. E. J. Newman, S. H. Strogatz, and D. J. Watts, Phys. Rev. Lett. 85, 5468 (2000).

[43] M. E. J. Newman, S. H. Strogatz, and D. J. Watts, Phys. Rev. E 64, 026118 (2001).

[44] A. Bunde and S. Havlin, in Fractals and Disordered Systems, edited by A. Bunde and S. Havlin (Springer, New York, 1996). 